\title{
Numerical investigation of Lagrangian coherent structures in steady rotation vortex shedding control
}

\author{
Ahsan Shehzad*, Tauseef-ur-Rehman**, Aamir Sohail***, Tehmina Ambreen** and \\ Muhammad Arsalan Anwar*** \\ * Mechanical Engineering Department, Pakistan Institute of Engineering and Applied Sciences, PIEAS, Nilore, 45650, Islamabad, Pakistan \\ ** School of Mechanical Engineering, Kyungpook National University, 80 Daehakro, Bukgu Daegu, 41566, South Korea \\ *** Mechanical Engineering Department, University of Engineering and Technology, Taxila, 47050, Pakistan \\ **Corresponding Author: tauseef@knu.ac.kr
}

Submitted : 10/07/2019

Revised : 20/07/2021

Accepted :01/08/2021

\begin{abstract}
In this paper, vortex shedding and suppression are numerically investigated as autonomous and nonautonomous dynamical systems, respectively. Lagrangian coherent structures (LCSs) are used as a numerical tool to analyze these systems. These structures are ridges of finite time Lyapunov exponent (FTLE), which act as material surfaces that are transport barriers within the flow. Initially, the utility of LCSs is explored for revealing the coherent structures of these systems. Finally, an active flow control method, steady rotation, is applied to the nonautonomous dynamical system with different speed ratios to mitigate vortex shedding magnitude. This will eventually turn the system into an autonomous system. Fixed saddle points, separation profiles essentially as unstable time variant manifolds attached to cylinder wall, and evolution of other unstable manifolds with variant speed ratios are analyzed with reference to LCSs. It is revealed that speed ratio of 2.1 fully suppresses the von Karman vortex street at Reynolds number of 100 and system turns into an autonomous dynamical system with fixed saddle points and time-invariant manifolds.
\end{abstract}

Keywords: Active flow control; Circular cylinder; Lagrangian coherent structures; Steady rotation control; Vortex shedding.

\section{INTRODUCTION}

The flow past bluff bodies have been put to computational and experimental research throughout the history of fluid mechanics. There are all kinds of strange behaviors of flow around bluff bodies. The creeping flow, pair of stable vortices, laminar vortex street, turbulent wake, and the transition of the laminar boundary layer to turbulent and turbulent vortex street are different characters of flow over bluff bodies, which are caused by the change in Reynolds number. The control of the flow field is also an active area. Two kinds of system are widely used from flow field perspective, autonomous and nonautonomous systems. Autonomous systems are one in which vortex shedding can be controlled as a self-dependent entity, whereas nonautonomous systems lack such kind of an intent. 
Autonomous systems often lead to the formation of Lagrangian coherent structures (LCSs). LCSs are static rotation zones existing in the flow field that interrupts an incoming flow and exhibits sufficient momentum.

Lagrangian coherent structures (LCSs) are important to consider from the engineering perspective. Such structures are powerful enough that they can damage the structure if not properly investigated. Marine structures like offshore rigs operate in a deep-sea environment, where vortex shedding occurs in a prominent manner at varying turbulence levels. During such phenomenon, undisturbed rotating patterns are formed in the flow that exhibits high level of energy. If such patterns formed near an offshore platform, they can damage the platform in a severe manner. So, identification of such coherent structures is essential for a safe working environment.

Chan et al. (2011) reviewed the recent advances in flow control particularly 3D forcing, local and global stability-based control. The authors revealed that unsteady vortex shedding can be stifled for the gap sizes ranges from $1 \mathrm{D}$ to $5 \mathrm{D}$. It was also noticed that unsteady wake suppression could be achieved by the rotation of cylinders in the opposite direction. Rockwood, Taira, and Green (2016) studied the wake behind the cylinder to explore the physics behind the complex vortex shedding. Lagrangian coherent structure (LCS) was used to formulate the investigation. In numerical results, as well as experimental results, it was noticed that Lagrangian saddle point remained attached to the surface of the cylinder until the separation of the vortex. An experimental study was carried out by Dol (2013), in which the author investigated the wake produced at Reynold's number 2000. Significant vortex changes were observed by the rotation of the cylinder.

The steady rotation of circular cylinder has also been utilized to control flow field around the cylinder. The speed ratio $(\lambda)$ is defined as the ratio of tangential velocity of the cylinder to free stream velocity of the fluid.(Barnes (2000), as well as some other recent researchers (Rodríguez et al. 2015; Jin and Dong 2016; Mao, Blackburn, and Sherwin 2015; Chandran, Janardhanan, and Menon 2018; Bovand et al. 2015), found that vortices were suppressed at $\lambda \geq 0.6$ for $\operatorname{Re} 50$. $\lambda \geq 1.5$ suppresses vortices for $\mathrm{Re}=2000$. An experimental study was conducted by Cicolin and Assi (2017), in which it concluded that the strength of vortex shedding got weakened by rotating the circular cylinder at speed ratios between 1 and 2.7. It was also reported that the speed ratio of 2 nearly suppresses the von Karman vortex street. In another study, the suppression of periodic vortex shedding was also studied experimentally by Dol, Kopp, and Martinuzzi (2008) by rotating the circular cylinder. They also concluded that vortices were almost suppressed at speed ratio 2. Both numerical and experimental investigations were conducted by Chan et al. (2011) to study the vortex shedding and suppression of vortex shedding for counter-rotating cylinders. A similar numerical study was conducted by Chan and Jameson (2010) to study the suppression of doublet like counter-rotating cylinders. Their results clearly revealed that when cylinders were rotated in counter directions, unsteady vortex could be completely suppressed.

The understanding of flow rheology plays a vital role in control methodology. One recent approach to understanding flow topology in a typical fluid dynamical system is to explain it with the help of time variant manifolds. FTLE field extracts these manifolds. The ridges of FTLE are called LCS (Haller 2001). Under the complex chaotic flow, there exist patterns of coherent structures that are sources of transport of passive fluid. LCS is a reliable tool to study this transport of passive fluid. Salman et al. (2007) studied the prediction of tracer transport by LCSs. Vortex was redefined objectively by Haller (2005) from the perspective of coherent structures. So, LCSs are of utmost importance in studying complex flows.

In the current research, the numerical study is conducted for the flow past a rotating circular cylinder at a fixed Reynolds number of 100 in order to determine the Lagrangian coherent structures (LCSs). Since the previous work regarding LCS is very limited and leaves a huge gap for the future research, the flow induces the vortex street at Reynolds number of 100 terms as Von Karman Vortex Street as stated by Mittal and Kumar (2003) and Meunier (2014). At such Reynolds number, the flow problem is 2D as 3D effects are negligible at low speeds (Barkley and Henderson 1996). The two-dimensionality of Von Karman Vortex Street motivates us to apply two-dimensional 
LCSs code. Since the rotation of the cylinder does not affect the dimensionality of flow, the idea of two-dimensional FTLE is utilized to study the flow field characteristics. Four different speed ratios are selected for the CFD analysis as $0.5,1,1.5$, and 2.1. The discrete velocity data is used to compute LCSs. LCSs provide the qualitative information of skeleton structures involved in the transport of fluid.

\section{METHODOLOGY}

\section{FINITE TIME LYAPUNOV EXPONENT (FTLE)}

Coherent structures form a skeleton that delineates the flow topology. These structures take an active part in the rheology of flow, helping passive fluid (requires no external energy source) to move, stretch, and compress in the physical domain. LCSs act locally as the most attracting, repelling, and shearing material surfaces. One way to find FTLE field is to find out the maximum stretching of perturbation placed close to a given point in a physical domain. Since FTLE is a computational parameter that is useful in order to determine the rate at which separation of the infinitesimally streamlines appears in a flow, the flow separation for such a technique can predict the location of LCSs during a flow. Consider a particle at $\mathrm{x}\left(t_{o}\right) \in \mathrm{D} \subset \mathbb{R}^{n}$ at an initial time $t_{o}$ when advected by flow field, after time $\mathrm{T}$ it moves to $\left(t_{o}\right) \mapsto \emptyset_{t_{o}}^{t_{o}+T}(x)$ and let the initial perturbation be $\delta x\left(t_{o}\right)$.

$$
\begin{aligned}
& y\left(t_{o}\right)=x\left(t_{o}\right)+\delta x\left(t_{o}\right) \\
& \delta x\left(t_{o}+T\right)=\frac{d \phi_{t_{o}}^{t_{o}+T}(x)}{d x} \delta x\left(t_{o}\right)+O\left(\left\|\delta x\left(t_{o}\right)\right\|^{2}\right)
\end{aligned}
$$

As initial perturbation is small, so $O\left(\left\|\delta x_{o}\right\|_{2}^{2}\right)$ can be neglected. $L_{2}$ gives the magnitude of this perturbation.

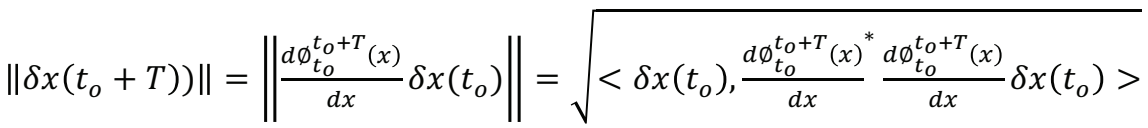

where $\frac{d \emptyset_{t_{o}}^{t_{o}+T}(x)^{*}}{d x}$ denotes the adjoint (transpose) of $\frac{d \emptyset_{t_{o}}^{t_{o}+T}(x)}{d x}$. The symmetric matrix

$\Delta\left(x, t_{o}, T\right)=\frac{d \phi_{t_{o}}^{t_{o}+T}(x)^{*}}{d x} \frac{d \phi_{t_{o}}^{t_{o}+T}(x)}{d x}$

Eq. 4 is referred to as the finite time version of Cauchy-Green deformation tensor. The motivation is to find out the maximum stretching of perturbation. This can be done only if the perturbation is aligned with the eigenvector corresponding to maximum eigenvalue of $\Delta\left(x, t_{o}, T\right)$. Therefore,

$$
\begin{aligned}
& \left.\max _{\delta x\left(t_{o}\right)} \| \delta x\left(t_{o}+T\right)\right) \|=\sqrt{\left.<\delta x\left(t_{o}\right), \lambda_{\max }\left(\Delta\left(x, t_{o}, T\right)\right) \delta x\left(t_{o}\right)\right\rangle} \\
& \left.=\sqrt{\lambda_{\max }\left(\Delta\left(x, t_{o}, T\right)\right)<\delta x\left(t_{o}\right), \delta x\left(t_{o}\right)>}=\sqrt{\lambda_{\max }\left(\Delta\left(x, t_{o}, T\right)\right)} \| \delta x\left(t_{o}\right)\right) \|
\end{aligned}
$$


$\sqrt{\lambda_{\max }\left(\Delta\left(x, t_{o}, T\right)\right)}$ is spectral norm of $\frac{d \emptyset_{t_{o}}^{t_{o}+T}(x)}{d x}$. The separation of two advected particles can be written in terms of Liapunov exponent

$\sigma_{t_{o}}^{T}(x)=\frac{1}{|T|} \ln \left(\sqrt{\lambda_{\max }\left(\Delta\left(x, t_{o}, T\right)\right)}\right.$

$\sigma_{t_{o}}^{T}(x)$ is a Finite Time version of Liapunov Exponent and is called FTLE field. It is a measure of the average maximum expansion of a pair of advected particles. If particles are integrated forward in time, the maximum expansion can be computed using FTLE field. But we are also interested to find out maximum compression of particles. One adopted way is to utilize the idea of backward integration, such that maximum compression of particles in forward time corresponds to maximum expansion in backward time. To address compression and expansion, manifolds are defined. Manifold here is defined in $\mathbb{R}^{n}$ as (n-1)-dimensional hypersurface $W$ that is smooth. The stable manifold $W^{s}\left(\mathrm{x}^{*}\right) \subset \mathrm{D}$ of a hyperbolic fixed point $\mathrm{x}^{*}$ is defined as

$W^{s}\left(\mathrm{x}^{*}\right)=\left\{\mathrm{x}_{\mathrm{o}} \subset \mathrm{D}: \lim _{\mathrm{t} \rightarrow \infty} \mathrm{x}\left(t ; \mathrm{x}_{\mathrm{o}}\right)=\mathrm{x}^{*}\right.$

The trajectories on either side of the stable manifold will diverge in forward time. Similarly, unstable manifold $W^{u s}\left(\mathrm{x}^{*}\right) \subset \mathrm{D}$ of a hyperbolic fixed point $\mathrm{x}^{*}$ is defined as

$W^{u s}\left(\mathrm{x}^{*}\right)=\left\{\mathrm{x}_{\mathrm{o}} \subset \mathrm{D}: \lim _{\mathrm{t} \rightarrow-\infty} \mathrm{x}\left(t ; \mathrm{x}_{\mathrm{o}}\right)=\mathrm{x}^{*}\right.$

The trajectories on either side of the unstable manifold will diverge in backward time. Both unstable and stable manifolds are invariant in autonomous systems.

\section{NUMERICAL PROCEDURE, RESULTS, AND DISCUSSIONS}

The precursor to apply LCSs methodology is to find out the velocity field of any dynamical system. This velocity field is utilized to integrate particles. In fluid mechanical systems, Computational Fluid Dynamics (CFD) tool is employed to study the velocity field. The velocity is generally a function that is dependent on both spatial and temporal coordinates. Once velocity field is obtained, it can be imported into the code. MATLAB code is used to compute FTLE with the following procedure:

- Create a structured grid of particles (LCS Grid).

- Read data file including mesh coordinates and corresponding velocities at the initial time step.

- Project velocities back to mesh coordinates and then interpolate velocities in the domain of LCS.

- Integrate particle positions in LCS domain with these interpolated velocities by using numerical integration. Euler's method of integration is usually used for this purpose.

- Integrate these integrated new positions using the velocities at the new time step, and repeat it until the integration time is reached.

- Calculate the deformation gradient for each particle and subsequently the FTLE.

- Extract ridges of FTLE, which are Computed Lagrangian Coherent Structures (LCSs).

The deformation gradient is calculated using the first order difference approximation. 


$$
\begin{aligned}
& \frac{d \Phi_{0}^{T}(x(i, j, k))}{d x}= \\
& {\left[\begin{array}{lll}
\frac{x(i+1, j, k)(T)-x(i-1, j, k)(T)}{x(i+1, j, k)(0)-x(i-1, j, k)(0)} & \frac{x(i, j+1, k)(T)-x(i, j-1, k)(T)}{y(i, j+1, k)(0)-y(i, j-1, k)(0)} & \frac{x(i, j, k+1)(T)-x(i, j, k-1)(T)}{z(i, j, k+1)(0)-z(i, j, k-1)(0)} \\
\frac{y(i+1, j, k)(T)-y(i-1, j, k)(T)}{x(i+1, j, k)(0)-x(i-1, j, k)(0)} & \frac{y(i, j+1, k)(T)-y(i, j-1, k)(T)}{y(i, j+1, k)(0)-y(i, j-1, k)(0)} & \frac{y(i, j, k+1)(T)-y(i, j, k-1)(T)}{z(i, j, k+1)(0)-z(i, j, k-1)(0)} \\
\frac{z(i+1, j, k)(T)-x(i-1, j, k)(T)}{x(i+1, j, k)(0)-x(i-1, j, k)(0)} & \frac{z(i, j+1, k)(T)-z(i, j-1, k)(T)}{y(i, j+1, k)(0)-y(i, j-1, k)(0)} & \frac{z(i, j, k+1)(T)-x(i, j, k-1)(T)}{z(i, j, k+1)(0)-z(i, j, k-1)(0)}
\end{array}\right]}
\end{aligned}
$$

Green Cauchy deformation tensor is then computed using the above equation. It is to be noted that Green Cauchy deformation tensor is a symmetric 3x3 matrix. Thus, it will have three Eigen values and corresponding Eigen vectors. The Eigen vector corresponding to the maximum Eigen value defines the maximum stretching direction. This information is utilized to calculate FTLE and plotted in Fig. 1. The darker red color represents the field with higher values. The ridges (higher values) extract LCSs.

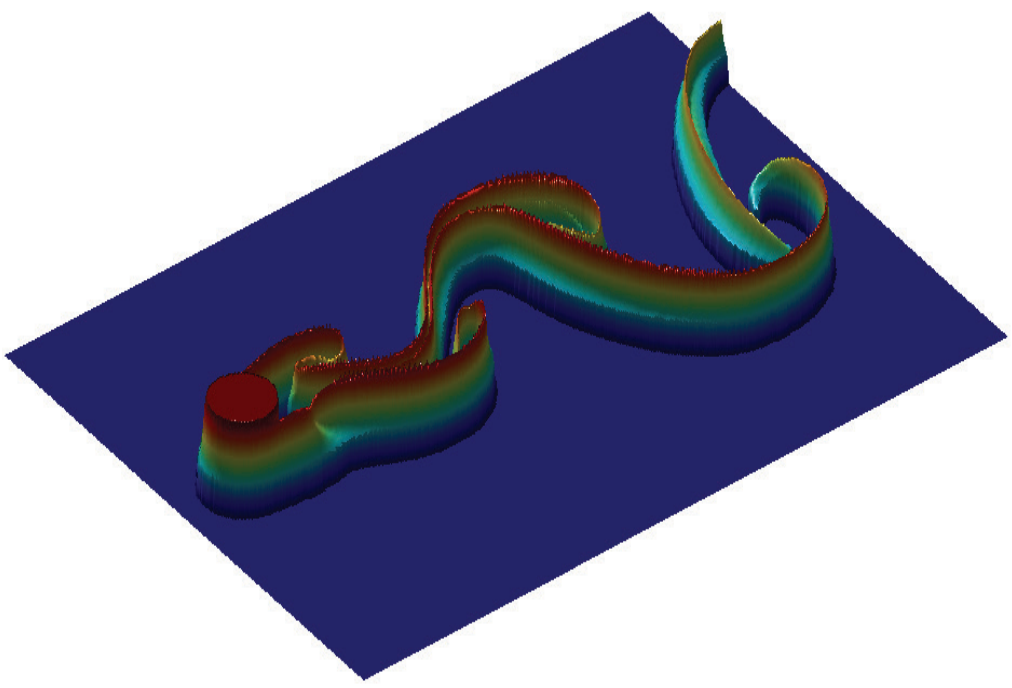

Figure 1. Depiction of FTLE field.

\section{VON KARMAN VORTEX STREET}

Two-dimensional fluid system is considered in Euclidean space with the region of the domain $D \in R^{2} \subset R^{n}, D=E / 0 \leq x_{1}{ }^{2}+y_{1}{ }^{2} \leq 0.25$ where $E=\{(x, y) \mid x \in[-5,15], y \in[-10,10]\}$. The flow field is computed using the commercial solver FLUENT 16.0. For a dimensionless analysis, the diameter is selected 
as 1 unit. Reynolds number of 100 equates the velocity $U=1 \mathrm{~m} / \mathrm{s}$, density $\rho=1 \mathrm{~kg} / \mathrm{m}^{3}$ and viscosity $\mu=0.01 \mathrm{~m}^{2} / \mathrm{s}$. The field is initialized with a steady solution of NS equations with Reynolds number of 100 . The transient analysis is done with time step $\Delta t=0.1 \mathrm{sec}$, such that the local maximum courant number does not exceed 2.70. The time step and grid are independent enough to capture the desired results. Pressure and velocity are coupled with PISO scheme. Momentum and pressures are spatially discretized with QUICK method, and secondorder implicit temporal formulation is used for transient formulation. Time histories of lift and drag coefficients are monitored and plotted in Fig. 2. Fast Fourier Transform of lift coefficient reveals the frequency of transverse oscillations.

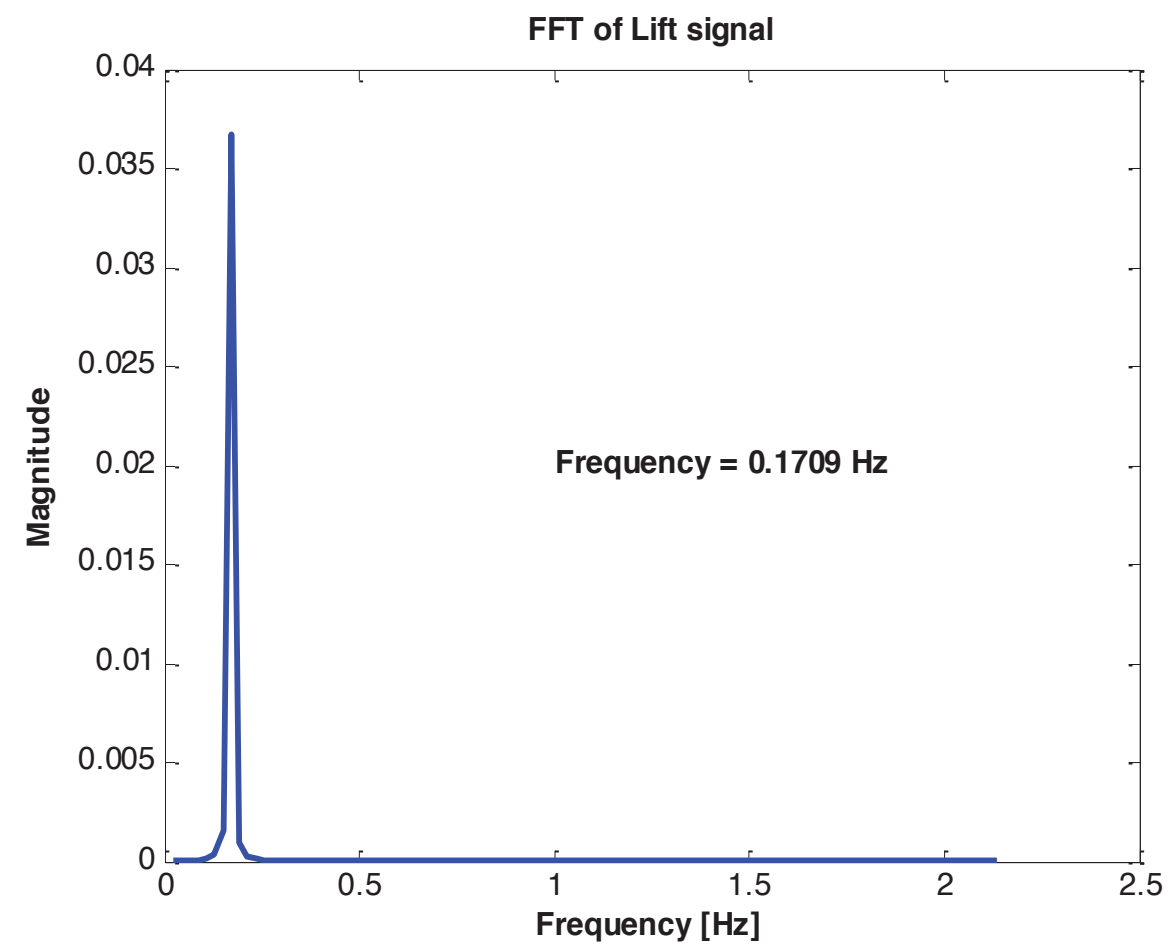

Figure 2. Illustration of FFT of Lift signal without rotation.

The frequency is calculated to be $f=0.1709 \mathrm{~Hz}$. The frequency is related to Strouhal number by the following relationship:

$S t=\frac{f D}{U}$

The nondimensionalization helps equate frequency with Strouhal number. Thus, Strouhal number is equal to 0.1709 . It is close to the value of 0.172 , provided by Mahir and Altaç (2008) with an error of approximately $0.6 \%$. The frequency elucidates the shedding of two periodic vortices. Both vortices rotate in opposite directions and shed downstream. The shedding of one vortex causes the formation of other vortex and the process continues. Velocity contours at one instant are shown in Fig. 3. 


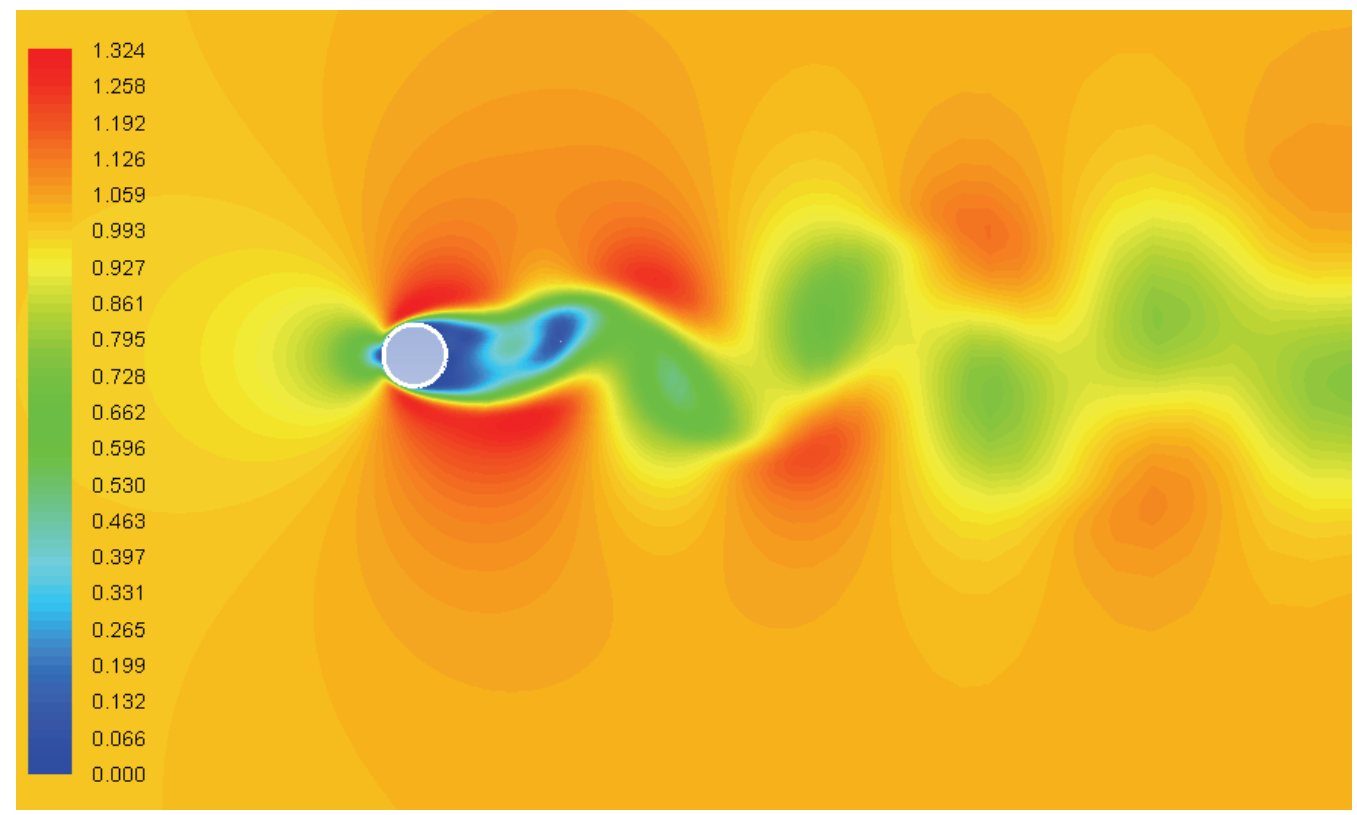

Figure 3. Depiction of velocity contours of von Karman vortex shedding.

Fig. 3 reveals that technical features like separation profile, separation point, wake dynamics, and fluid advection are not evident from velocity information. FTLE approach is utilized to diagnose underlying structures. Velocity at each time step is monitored and used to calculate FTLE field. The FTLE grid spacing is selected in such a way that each unit of domain length is divided into 100 equal parts. The realistic computation of LCSs requires small grid spacing (Haller 2005). The unstable manifolds stretch the tracer group tangent to the manifold. When this manifold is attached to the solid wall as in the case of a circular cylinder, it acts as a separatrix. Thus, particles are drawn towards it and flee tangently to it without crossing it. This manifold separates the recirculation zone of eddy with shear layer. The separation point and separation profile thus correspond to this manifold. This is evident from Fig. 4, where U1 and U2 manifolds correspond with separation profiles. Fig. 5 reflects the unstable manifolds for a continuous flow phenomenon at the cylinder wall, since vortex shedding appears after fixed intervals and must be observed carefully regarding formation of unstable manifolds.

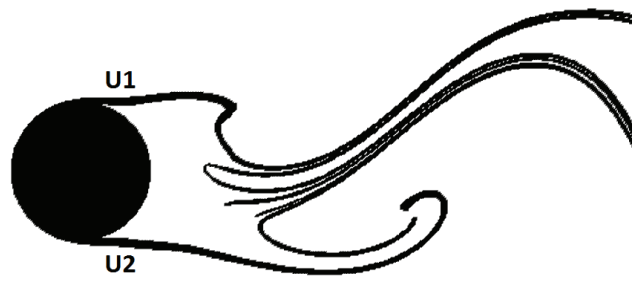

Figure 4. Separation profiles associated with unstable manifolds U1 and U2. 


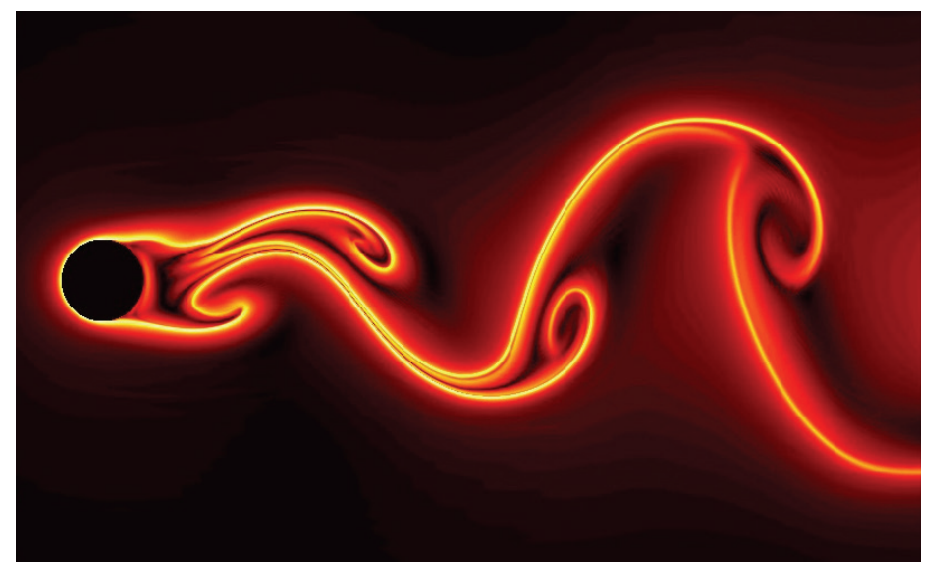

Figure 5. Illustration of instantaneous unstable manifolds.

The complete evolution of unstable manifolds is presented in Fig. 6.
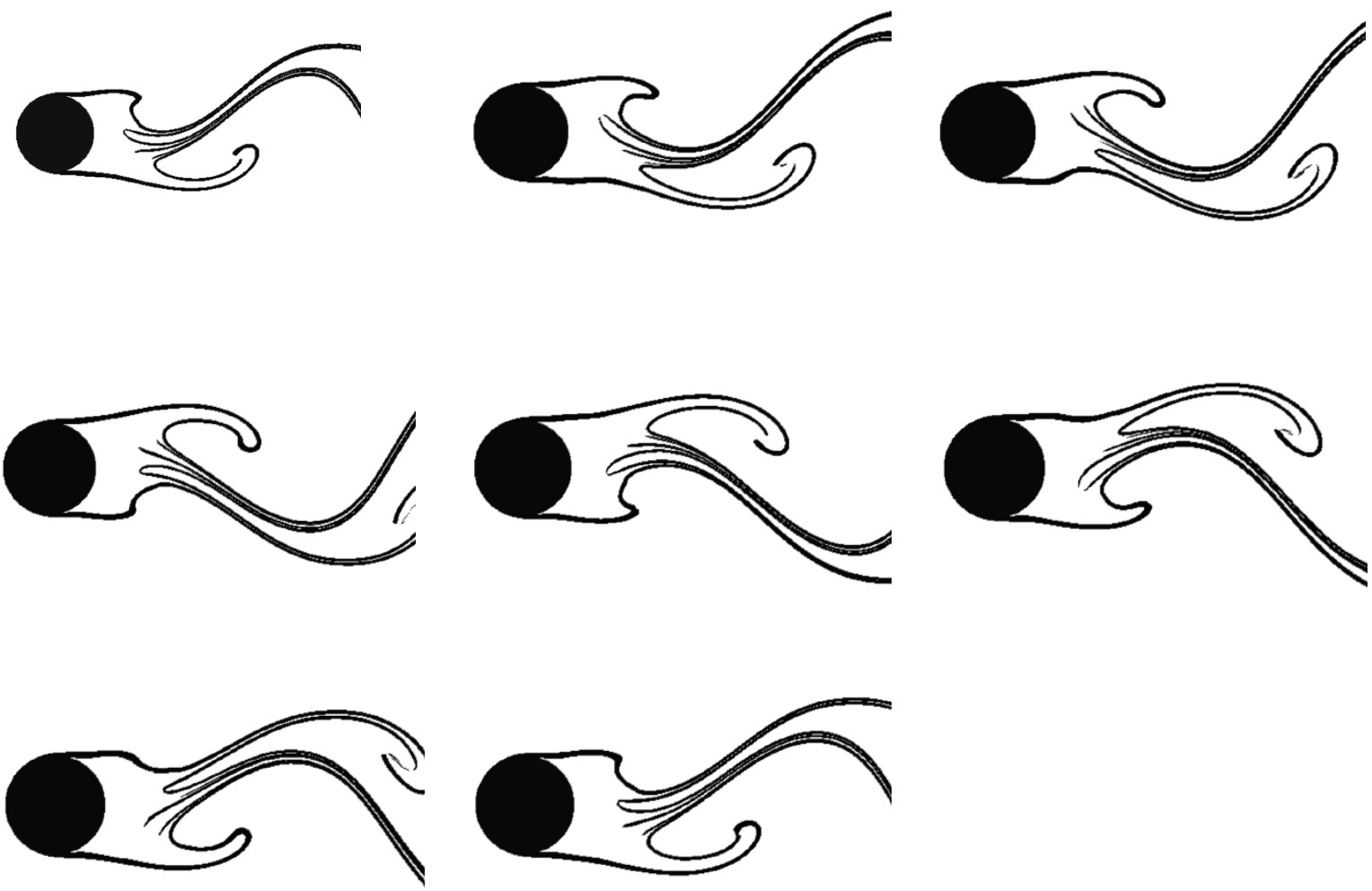

Figure 6. Evolution of unstable manifolds at integration time 9 Sec. 


\section{SUPPRESSION OF VORTEX SHEDDING}

The steady rotation is chosen here as an active flow control method for the suppression of these vortices. The cylinder is rotated at different steady speed ratios, and the velocity data is recorded. The speed ratio is defined as

$$
\lambda=\frac{r \Omega}{U}
$$

where $r$ is the radius of a circular cylinder, and $U$ is the free stream velocity. $\Omega$ is the angular frequency in $\mathrm{rad} / \mathrm{sec}$. The cylinder is rotated at four speed ratios and magnitude of oscillations in lift is recorded. The magnitude recorded is defined as

$$
\left|C l_{p-p}\right|=\left|C l_{\max }-C l_{\min }\right|
$$

Table 1. Value of $\left|C l_{p-p}\right|$ at various speed ratios.

\begin{tabular}{|c|c|}
\hline Speed ratio & Magnitude $\left|C l_{p-p}\right|$ \\
\hline 0.0 & 0.5104 \\
\hline 0.5 & 0.5401 \\
\hline 1.0 & 0.5625 \\
\hline 1.5 & 0.4442 \\
\hline 2.1 & 0.0053 \\
\hline
\end{tabular}

It is evident from Table 1 that the strength of vortices mitigates for higher values of speed ratios. The value of speed ratio 2.1 completely suppresses unsteady vortex shedding. It must be noted that the cylinder is rotated counterclockwise; therefore, Magnus forces decrease the lift and even generate negative lift. If it had rotated in the clockwise direction, the Magnus forces would have generated positive lifts. The physics, however, does not change in both cases. Therefore, the results of anticlockwise rotation can directly correspond to the results of clockwise rotation. The lift graph for different speed ratios introduced at different instants is plotted in Fig. 7. The lift oscillation pattern and values are consistent to the published results of Paramane and Sharma (2009) and Ikhtiar et al. (2016). The velocity contours for the flow past a cylinder at speed ratio of 2.1 are shown in Fig. 8. As a result of high counterclockwise rotation, the shear layer (that rolls round a cylinder) stretches over a cylinder in the downstream flow. It indicates that, at $\lambda=2.1$, no shedding of vortices will appear as indicated by a straight line in Fig. 7 . 


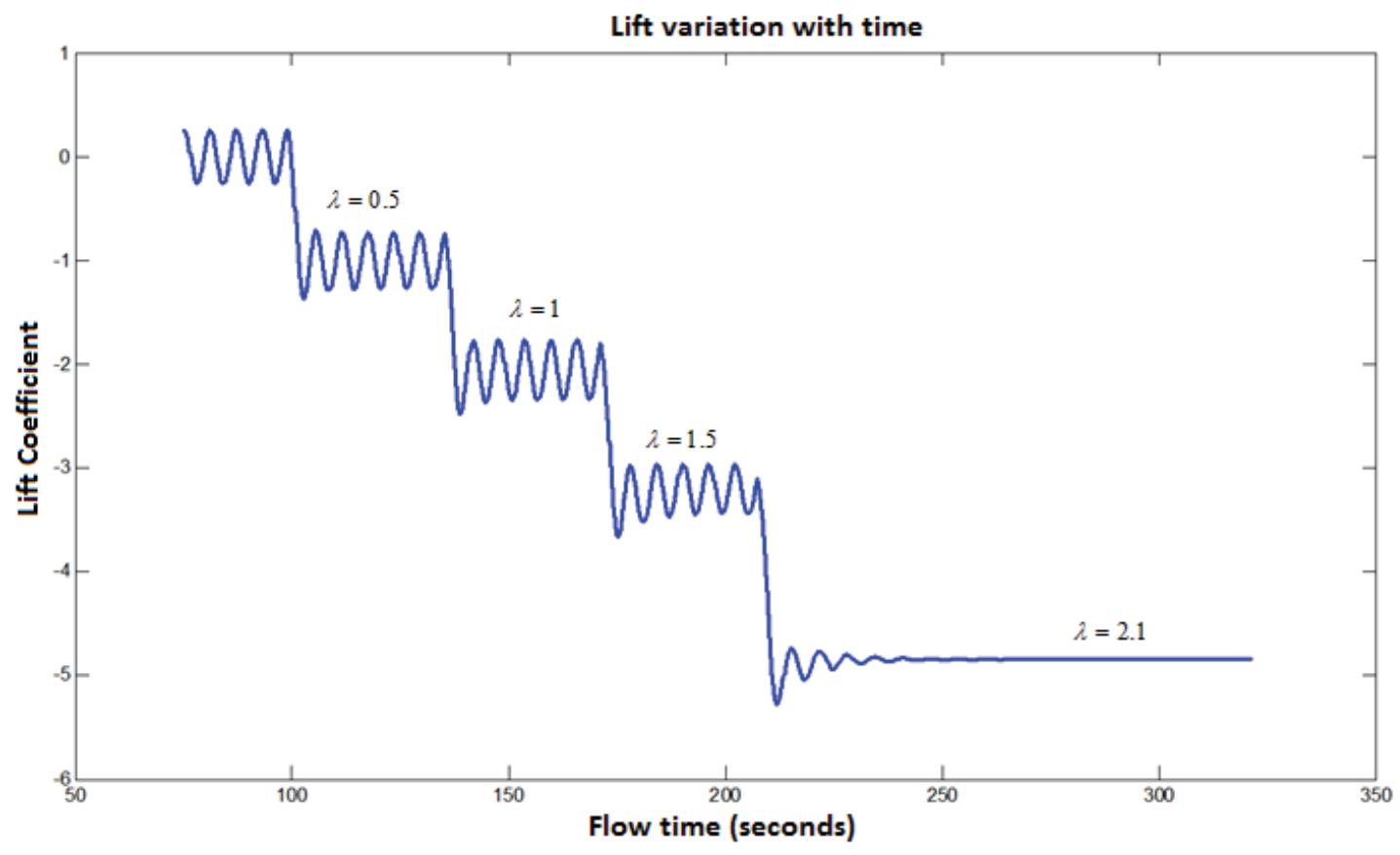

Figure 7. Lift variation with time at various speeds.

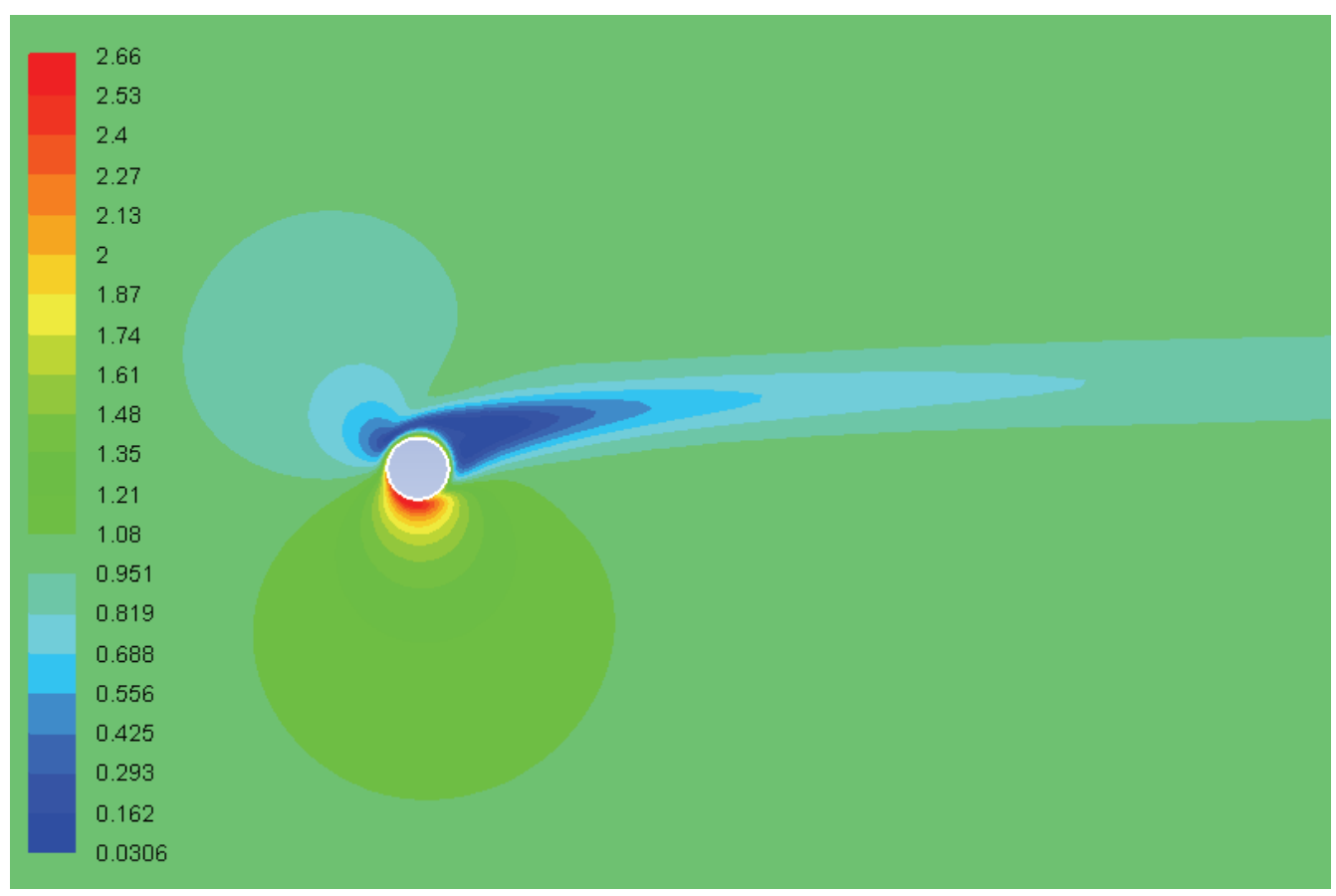

Figure 8. Velocity contours of the autonomous system at $\lambda=2.1$ 
From the perspective of unstable manifolds, this suppression is shown in Fig. 9. U1 and U2 are only unstable manifolds that are attached to the solid wall. Therefore, they are most affected by the rotation of the cylinder wall. Fig. 9 demonstrates that $\mathrm{U} 1$ and $\mathrm{U} 2$ immediately respond to rotation of cylinder, while information has not yet been passed to all other manifolds. All other manifolds behave exactly in the same manner as if the cylinder is not rotated. The particles attached to the wall acquire a velocity of the rotation, and the viscosity stratifies the velocity as distance normal to the wall increases. This is the way to pass information to the flow field. One clear consequence is that both $\mathrm{U} 1$ and $\mathrm{U} 2$ manifolds become curved in a circular fashion in the direction of rotation (counterclockwise here). They continue to move until they both combine to form one manifold U12. Fig. 9 (a-e) shows the evolution of this integration. U3 and U4 manifolds are defined here after the merger of U1 and U2 into U12. Two manifolds are integrated into one manifold at the upstream and shed to the downstream. The upstream fluid pushes U3 and U4 manifolds and bends them, causing them to nearly merge into one another. This process continues, and, at the same time, U12 manifold grows until fig h, where U3 and U4 separate from one another again. The U5 manifold also appears. The U5 manifold separates the region above it, indicating the recirculation or a weakly oscillating vortex and region below it, which is backward (reverse) flow as the manifestation of wall rotation. U4 in Fig. 9 depicts that it causes the shedding of the weakly oscillating vortex. U5 manifold periodically appears and disappears, and U45 periodically sheds vortex, and the process continues until the system damps out the weak oscillations of the vortex, and it becomes a steady stable single vortex. The system transient state turns into a steady state. Fixed saddle point appears in the domain.

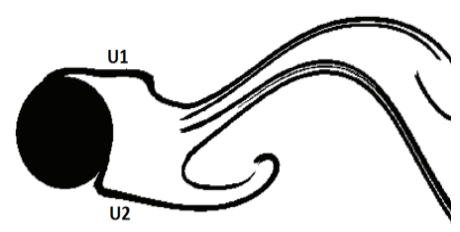

a

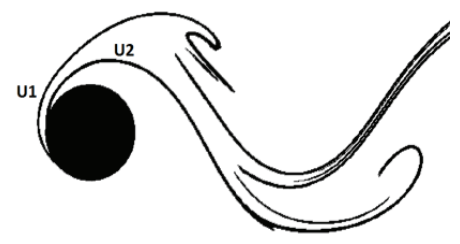

d

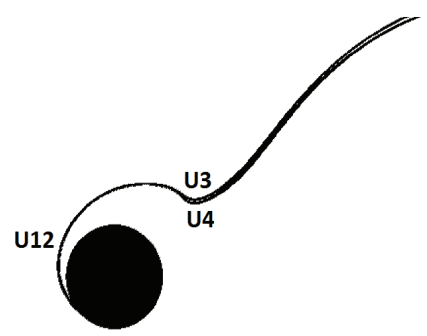

g

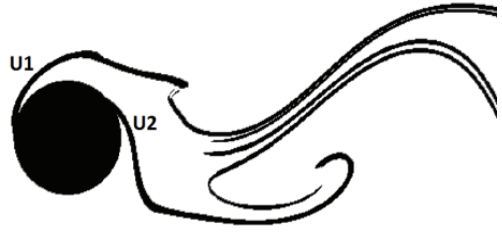

b

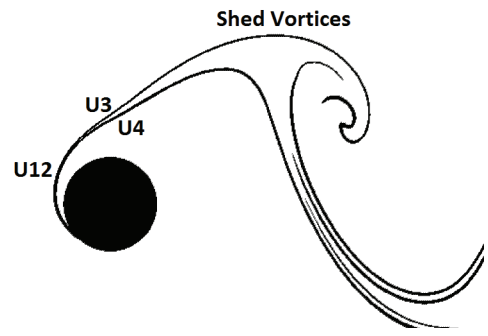

e

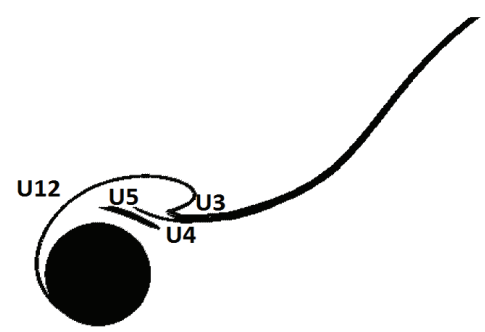

h

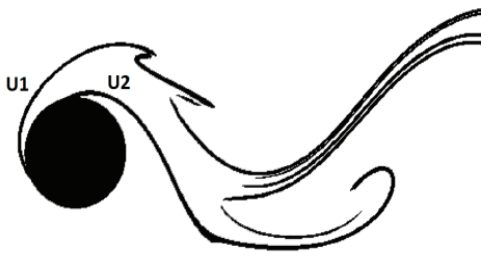

c

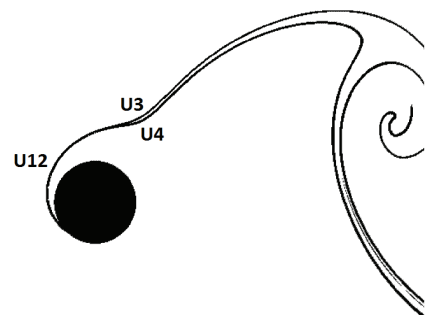

f

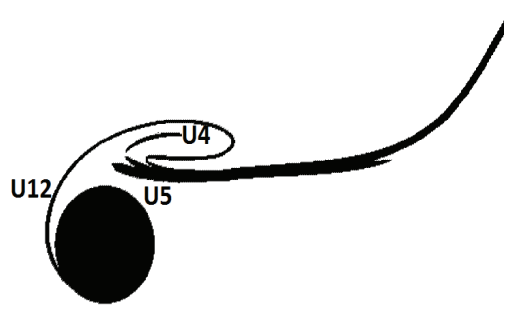




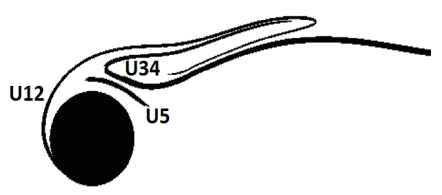

$\mathrm{j}$

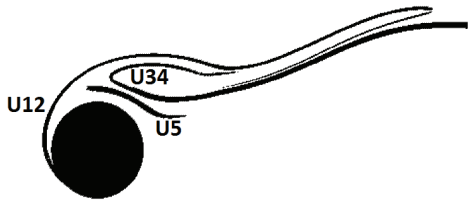

$\mathrm{k}$

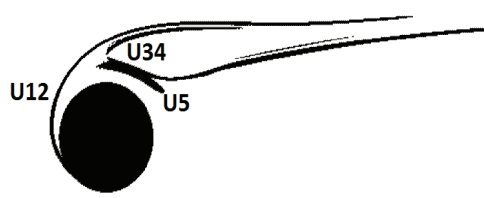

1

Figure 9. Evolution of unstable manifold for speed ratio 2.1.

Stable and unstable manifolds are overlaid in Fig. 10. Intersection of these manifolds spots the fixed saddle point. The particles follow streamlines in this system.

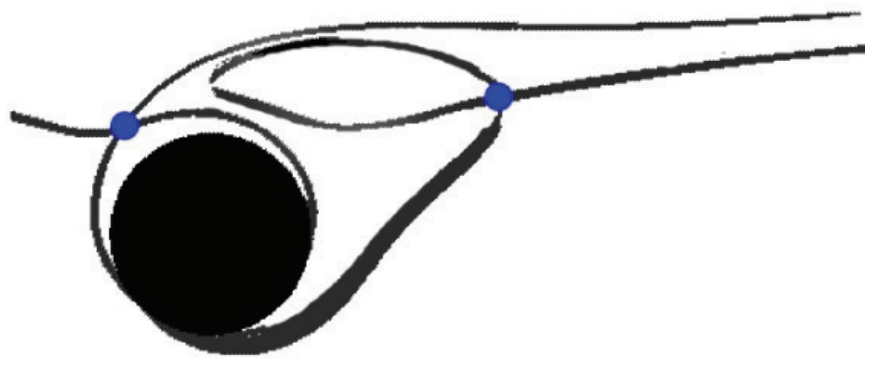

(a) The intersection of manifolds at two saddle point.

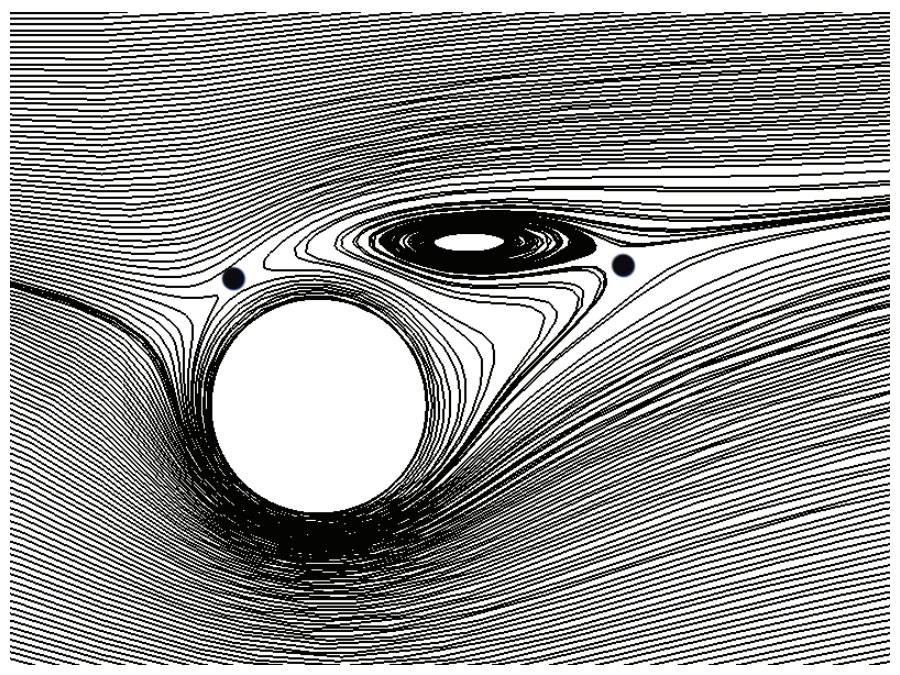

(b) Corresponding streamlines acting as trajectories of particles.

Figure 10. Illustration of an autonomous system. 


\section{CONCLUSION}

- Vortex shedding and suppression are analyzed with reference to Lagrangian coherent structures. Vortex Street is controlled with varying speed ratios. The speed ratio of 2.1 completely suppressed oscillations and is in complete agreement to the previous research work. The system is shown to have turned into an autonomous system.

- It is also demonstrated that separation profiles are associated with only unstable manifolds. The separation point moved back and forth on the cylinder wall and is depicted by unstable manifolds. Thus, these manifolds are adequate in explaining vortex shedding.

- It is further revealed that the suppression of vortices caused two fixed saddle points to appear in a steady state. The overlaid stable and unstable manifolds revealed local recirculation zone. Only one stable vortex was confined within the boundaries of LCSs.

- The integration of the equation of streamline computed the trajectories of fluid particles. Hence, the unstable manifolds, in the considered non-autonomous system, and both stable and unstable manifolds in the autonomous system are required to understand the flow physics as mentioned in the literature.

\section{REFERENCES}

Barkley, Dwight, and Ronald D Henderson. 1996. 'Three-dimensional Floquet stability analysis of the wake of a circular cylinder', Journal of Fluid Mechanics, 322: 215-41.

Barnes, FH. 2000. 'Vortex shedding in the wake of a rotating circular cylinder at low Reynolds numbers', Journal of Physics D: Applied Physics, 33: L141.

Bovand, M, Saman Rashidi, M Dehghan, Javad Abolfazli Esfahani, and MS Valipour. 2015. 'Control of wake and vortex shedding behind a porous circular obstacle by exerting an external magnetic field', Journal of Magnetism and Magnetic Materials, 385: 198-206.

Chan, Andre S, Peter A Dewey, Antony Jameson, Chunlei Liang, and Alexander J Smits. 2011. 'Vortex suppression and drag reduction in the wake of counter-rotating cylinders', Journal of Fluid Mechanics, 679: $343-82$.

Chan, Andre S, and Antony Jameson. 2010. 'Suppression of the unsteady vortex wakes of a circular cylinder pair by a doublet-like counter-rotation', International journal for numerical methods in fluids, 63: 22-39.

Chandran, Vidya, Sheeja Janardhanan, and Varun Menon. 2018. 'Numerical Study on the Influence of Mass and Stiffness Ratios on the Vortex Induced Motion of an Elastically Mounted Cylinder for Harnessing Power', Energies, 11: 2580.

Cicolin, Murilo Marangon, and GRS Assi. 2017. 'Experiments with flexible shrouds to reduce the vortexinduced vibration of a cylinder with low mass and damping', Applied Ocean Research, 65: 290-301.

Dol, Sharul S. 2013. 'Weakened Vortex Shedding from a Rotating Cylinder', International Journal of Mechanical, Industrial Science and Engineering, 7: 330-37.

Dol, Sharul Sham, Gregory A Kopp, and Robert J Martinuzzi. 2008. 'The suppression of periodic vortex shedding from a rotating circular cylinder', Journal of Wind Engineering and Industrial Aerodynamics, 96: 1164-84.

Haller, George. 2001. 'Distinguished material surfaces and coherent structures in three-dimensional fluid flows', Physica D: Nonlinear Phenomena, 149: 248-77. 2005. 'An objective definition of a vortex', Journal of Fluid Mechanics, 525: 1-26. 
Ikhtiar, Usman, S Manzoor, NA Sheikh, and Muzaffar Ali. 2016. 'Free stream flow and forced convection heat transfer around a rotating circular cylinder subjected to a single gust impulse', International Journal of Heat and Mass Transfer, 99: 851-61.

Jin, Yiming, and Ping Dong. 2016. 'A novel wake oscillator model for simulation of cross-flow vortex induced vibrations of a circular cylinder close to a plane boundary', Ocean Engineering, 117: 57-62.

Mahir, Necati, and Zekeriya Altaç. 2008. 'Numerical investigation of convective heat transfer in unsteady flow past two cylinders in tandem arrangements', International Journal of Heat and Fluid Flow, 29: 1309-18.

Mao, X, Hugh Maurice Blackburn, and Spencer John Sherwin. 2015. 'Nonlinear optimal suppression of vortex shedding from a circular cylinder', Journal of Fluid Mechanics, 775: 241-65.

Meunier, Patrice. 2014. 'Laminar-Turbulent Transition in Stratified Wakes.' in, Experimental and Computational Fluid Mechanics (Springer).

Mittal, Sanjay, and Bhaskar Kumar. 2003. 'Flow past a rotating cylinder', Journal of Fluid Mechanics, 476: 303-34.

Paramane, Sachin B, and Atul Sharma. 2009. 'Numerical investigation of heat and fluid flow across a rotating circular cylinder maintained at constant temperature in 2-D laminar flow regime', International Journal of Heat and Mass Transfer, 52: 3205-16.

Rockwood, Matthew P, Kunihiko Taira, and Melissa A Green. 2016. 'Detecting vortex formation and shedding in cylinder wakes using Lagrangian coherent structures', AIAA Journal, 55: 15-23.

Rodríguez, I, O Lehmkuhl, J Chiva, Ricard Borrell, and A Oliva. 2015. 'On the flow past a circular cylinder from critical to super-critical Reynolds numbers: Wake topology and vortex shedding', International Journal of Heat and Fluid Flow, 55: 91-103.

Salman, Hayder, Jan S Hesthaven, Tim Warburton, and George Haller. 2007. 'Predicting transport by Lagrangian coherent structures with a high-order method', Theoretical and Computational Fluid Dynamics, 21: 39-58. 